\title{
Questionnaire Survey from the 1st Kurume University Inflammatory Bowel Disease Center Educational Lecture
}

\author{
HIROSHI YAMASAKI ${ }^{1,2,3}$, TETSUSHI KINUGASA ${ }^{4}$, SHOKO IWASAKI $^{5}$, SHINICHIRO YOSHIOKA $^{1,2}$, \\ TATSUKI MIZUOCHI ${ }^{6}$, MIKIO ISHIBASHI ${ }^{7}, \mathrm{KEIKO} \mathrm{NAGATSUKA}^{8}, \mathrm{RYOSUKE} \mathrm{YAMAUCHI}^{1,2}$, \\ NORIKO ISHIBASHI ${ }^{9}$, TOSHIHIRO ARAKI ${ }^{1}$, ATUSHI MORI ${ }^{1,2}$, YOSHITO AKAGI $^{4}$, \\ KEIICHI MITSUYAMA ${ }^{1,2}$ AND TAKUJI TORIMURA ${ }^{1}$, \\ FOR THE KURUME UNIVERSITY IBD SUPPORT TEAM
}

\footnotetext{
${ }^{1}$ Division of Gastroenterology, Department of Medicine, Kurume University School of Medicine, Kurume 830-0011, Japan ${ }^{2}$ Inflammatory Bowel Disease Center, Division of Gastroenterology, Department of Medicine, Kurume University School of Medicine, Kurume 830-0011, Japan

${ }^{3}$ Hakuai Hospital, Kurume 839-0863, Japan

${ }^{4}$ Department of Surgery, Kurume University School of Medicine, Kurume 830-0011, Japan

${ }^{5}$ Department of Nutrition, Kurume University Hospital, Kurume 830-0011, Japan

${ }^{6}$ Department of Pediatrics and Child Health, Kurume University School of Medicine, Kurume 830-0011, Japan

${ }^{7}$ Department of Pharmacy, Kurume University Hospital, Kurume 830-0011, Japan

${ }^{8}$ Department of Nursing, Kurume University Hospital, Kurume 830-0011, Japan

${ }^{9}$ Medical Collaboration Center, Kurume University Hospital, Kurume 830-0011, Japan
}

Received 20 March 2018, accepted 13 July 2018

J-STAGE advance publication 9 August 2019

Edited by OSAMU TSURUTA

\begin{abstract}
Summary: Introduction: The number of inflammatory bowel disease (IBD) patients is increasing steadily in Japan, and it is expected that patient groups and patient education will improve the quality of life of patients and IBD care. The 1st Kurume University IBD Center educational lecture was held and a questionnaire survey was administered at this lecture.

Methods: We asked 78 participants to answer a questionnaire survey on the occasion of the 1st Kurume University IBD Center educational lecture.

Results: We obtained responses from 56 (71.8\%) participants; 31 (55.4\%) had IBD [21 (37.4\%) had ulcerative colitis (UC) and 10 (17.9\%) had Crohn's disease (CD)]. Most participants were female (37, 66\%). The age range with the highest number of participants was 40 to $69(27,48.2 \%)$. Most had heard about this educational lecture through "notification by the patient's doctor" 23 (41.1\%). A total of $30(53.6 \%)$ of participants answered "good" about the lecture content, while $50(89.7 \%)$ of participants answered "very good" and "good" about the impression of this lecture. Meanwhile, $10(32.3 \%)$ of patients were interested in patient groups. The percentage of patients who were interested in patient groups was higher in patients with CD $4(66.7 \%)$ than those with UC $2(33.3 \%)$.

Conclusion: We held the 1st Kurume University IBD center educational lecture. Further studies are needed to assess whether educational lectures and/or patient groups can improve patients' quality of life (QOL) and IBD care in our hospital.
\end{abstract}

Key words questionnaire survey, IBD, the multidisciplinary team, QOL

Corresponding Author: Hiroshi Yamasaki, MD \& PhD, Division of Gastroenterology, Department of Medicine, Kurume University School of Medicine, 67 Asahi-machi, Kurume 830-0011, Japan. Tel: +81-942-31-7561, Fax: +81-942-34-2623, E-mail: yamasaki_hiroshi@kurume-u.ac.jp

Abbreviations: CD, Crohn's Disease; IBD, inflammatory bowel disease; ICHOM, International Consortium for Health Outcomes Measurement; QOL, quality of life; UC, ulcerative colitis. 
The number of inflammatory bowel disease (IBD) patients is increasing steadily in Japan [1]. While the etiology is unknown, the therapeutic goal is not only to improve symptoms but also to improve the quality of life (QOL) of IBD patients. Meanwhile, it is also expected that patient groups and patient educational meetings will improve patient QOL and quality of care in institutions [2]. On June 25, 2017, the 1st Kurume University IBD Center educational lecture was held (Table 1). The number of participants was limited to 100 people on a first-come, first-served basis because of the capacity of the venue. We then asked 78 participants to fill out a paper questionnaire survey. This research was approved by Kurume University's ethics committee. We obtained responses to the questionnaire from $56(71.8 \%)$ of the attendees of the educational lecture. Table 2 shows the demographic data of the participants. There was a higher proportion of women [37 (66\%)] compared with men [19 (34\%)]. The greatest number of participants (27) were in their 40 's to 60's (48.2\%). The number of participants who were patients themselves was $31(55.4 \%)$, where 21 (37.5\%) had ulcerative colitis (UC) and $10(17.9 \%)$ had Crohn's Disease (CD). The number of participants who lived in Kurume city was 24 (42.9\%), while 32 $(57.1 \%)$ lived in another region or did not give a response. Patients at our hospital made up 25 (44.6\%) of the participants, while $31(55.4 \%)$ were from other hospitals or did not give a response. There were 311 IBD patients registered at Kurume University based on specific disease documents in 2016, and participants this time were $8 \%$ (25) of registered patients.
While UC was the most frequent of all diseases in terms of the number of participants by disease, the proportion of UC among the registered patients at our hospital by disease was $15(6.5 \%)$ for UC and $8(10 \%)$ for $\mathrm{CD}$, indicating that $\mathrm{CD}$ is the more frequent. As regards publicity for the event, first we directly told patients visiting Kurume university hospital about this lecture and posted information on our website. Notifications to patients at other relevant hospitals were not made this time. Second, we asked Kurume city to become an official sponsor of the lecture. Thereafter, we promoted the lecture by posters and leaflets at two major terminal stations in Kurume city, Kurume city plaza and Kurume health center and published information in Kurume city's public magazine. And finally, we asked the newspaper company to be a sponsor, and conference information was similarly published in local newspapers and an IBD patient specialty magazine (CCJAPAN). The most common way that participants became aware of this educational lecture was 'notification from the patient's doctor 23 $(41.1 \%)$, followed by posters and leaflets $9(16.1 \%)$ and recommendation by family members and friends $9(16.1 \%)$. Regarding the contents of the lecture, 30 (53.6\%) of participants answered 'good' about the overall lecture content (Table 3). Lecture 1 had the highest number of 'good' responses at 38 (67.9\%). A total of $10(32.3 \%)$ of patients participating in this lecture were interested in patient groups (Table 4). Regarding admission, 6 (19.4\%) of patients wanted to join a patient group while $8(25.8 \%)$ did not. The percentage of patients with $\mathrm{CD}$ who were interested in

TABLE 1.

The 1st Kurume university Inflammatory Bowel disease (IBD) center extension lecture to the public $\sim$ Come and Meet $\sim$

Date: June 25 (Sun.), $2017 \quad$ Venue: Kurume City Plaza

Supported by Kurume University IBD support team

Program

$<13: 30-14: 05>$ Special Lecture 1 I want to know about IBD

Dr. Keiichi Mitsuyama, Kurume University Hospital Inflammatory Bowel disease center

$<$ 14:20-15:10> Panel discussion - The multidisciplinary, patient centered approach to IBD care

$<15: 10-15: 25>$

Special Lecture 2

Specific medical expenses (designated intractable disease) application

$<15: 45-16: 30>$ Talk to Experts 
participating in the patient group $4(66.7 \%)$ was higher than those patients with UC $2(33.3 \%)$. The lecture participation rate of patients in Kurume University hospital was not high. Some patients were not notified by their doctors, so we realized that it was important to notify patients earlier next time. It was also considered important to notify patients at related hospitals. More UC participants were male than female, whereas there were more female $\mathrm{CD}$ participants than males. In contrast, the proportion of male patients with $\mathrm{CD}$ is high in Japan [1], and it is thought that the participation rate of female patients with $\mathrm{CD}$ was high even from that point of view. With regards to the ratio of men and women, a search of reports on the internet showed differences in the ratio of sexes between reports [3]. More $\mathrm{CD}$ patients wanted to join a patient group than UC patients. A previous report has suggested that CD

TABLE 2.

Demographics of educational lecture

\begin{tabular}{lc}
\hline & Total \\
& $(\mathrm{n}=78)$ \\
\hline Female participants, n (\%) & $37(66.1)$ \\
& \\
Age, n (\%) & $0(0)$ \\
10's to 20's & $8(14.3)$ \\
20's to 30's & $7(12.5)$ \\
30's to 40's & $12(21.4)$ \\
40's to 50's & $15(26.8)$ \\
50's to 60's & $10(17.9)$ \\
60's to 70's & $3(5.4)$ \\
70's to 80's & $1(1.8)$ \\
Blank & \\
Disease, n (\%) & $10(17.9)$ \\
CD & $21(37.5)$ \\
UC & $5(8.9)$ \\
Other & $20(35.7)$ \\
Blank & \\
Region, n (\%) & \\
Kurume city & $24(42.9)$ \\
Other & $23(41.1)$ \\
Blank & $9(16.1)$ \\
Kurume university patient, n (\%) & $1(1.8)$ \\
Yes & \\
No & \\
Blank & $23(41.1)$ \\
The way of knowing about this lecture, n $(\%)$ & $9(16.1)$ \\
The notification from the patient's doctor & $3(5.4)$ \\
Posters and leaflets & \\
Recommended by family members and friends & \\
CCJAPAN & $25(44.6)$ \\
Homepage & \\
New paper & \\
Other & \\
Blank & \\
\hline
\end{tabular}

patients generally have higher treatment adherence than UC patients [2]. The reason may lie in the fact that surgery is often necessary due to the various complications that can occur with $\mathrm{CD}$, therefore $\mathrm{CD}$ patients are more disabled than UC patients in daily life [4]. As a result of this background, it seems that the needs for $\mathrm{CD}$ patient groups may be higher. Educational lectures are being held at IBD centers with high expertise worldwide [5]. Previous studies of patient education have reported mixed effects, with some showing certain effects on improving QOL [6] and others showing no significant effects [7]. In these studies, it was possible that QOL evaluation of IBD patients may have been problematic. Therefore, we will plan to evaluate outcomes of educational lectures using the International Consortium for Health Outcomes Measurement (ICHOM) which is the defining global

TABLE 3.

Evaluation on content of lecture

\begin{tabular}{lc}
\hline & $\begin{array}{c}\text { Total } \\
(\mathrm{n}=78)\end{array}$ \\
\hline $\begin{array}{l}\text { The contents of the lecture, } \mathrm{n}(\%) \\
\text { Good }\end{array}$ & $42(53.6)$ \\
Blank & $36(46.4)$ \\
& \\
The overall impression of the lecture, $\mathrm{n}(\%)$ & \\
Very good & $26(46.4)$ \\
Good & $24(42.9)$ \\
Average & $0(0)$ \\
Below average & $1(1.8)$ \\
Other & $0(0)$ \\
Blank & $5(8.9)$ \\
\hline
\end{tabular}

TABLE 4.

Participation in the patient group

\begin{tabular}{lc}
\hline & Patients \\
\hline Interested in patient group, $\mathrm{n}(\%)$ & \\
Yes & $10(32.3)$ \\
No & $6(19.4)$ \\
Blank & $15(48.4)$ \\
Admission of patient group, n (\%) & \\
Yes & $6(19.4)$ \\
No & $8(25.8)$ \\
Blank & $17(54.8)$ \\
Admission of patient group (only interested & \\
in patient group), $\mathrm{n}(\%)$ & $6(60)$ \\
Yes & $2(20)$ \\
No & $2(20)$ \\
Blank & \\
Admission of patient group, $\mathrm{n}(\%)$ & \\
UC & $2(33.3)$ \\
CD & $4(66.7)$ \\
\hline
\end{tabular}


standard set of outcome measures that really matter to patients for the most relevant medical conditions [8]. Although our research is an observational study and we cannot state the effectiveness of patient education this time, at least it was found that the level of interest of IBD patients is high. In addition, there are also reports that showed that activation of multiple factors by participating in a patient group are effective for improving the adherence to medications [2]. In our study, we found that $\mathrm{CD}$ is of particular interest for patient association. In this survey, the number of subjects was small. There was also a gender difference. Therefore, it is thought that the matching of age and sex will also be necessary when conducting cohort studies and intervention studies in the future. In conclusion, we will further examine the questionnaire contents so as to improve future educational lectures. Furthermore, cohort studies including a follow-up study and intervention studies will be considered and performed with reference to this research.

COMPETING INTERESTS: The authors have declared that no competing interests exist in this research.

ACKNOWLEDGEMENTS: This research was supported partly by the IBD support team in Kurume University Hospital. Members in the Kurume University IBD support team are Hiroshi Yamasaki, Toshihiro Araki, Atushi Mori, Ryosuke Yamauchi, Syuhei Fukunaga, Kotaro Kuwaki, Shinichiro Yoshioka, Hidetshi Takeatsu, Keiichi Mitsuyama (Division of Gastroenterology), Tetsushi Kinugasa, Yoshito Akagi (Department of Surgery), Keisuke Eda, Takagi Takayoshi, Tadahiro Yanagi, Tatsuki Mizuochi (Department of Pediatrics), Yuuki Uchida, Yasushi Yamada, Shoko Iwasaki (Department of Nutrition), Tomoka Fujitake, Wako Nakamura, Keiko Nagatsuka, Rumi Kubo, Rumiko Sonoda, Miho Kugishima, Maaya Matsumoto, Chiyomi Shimokawa, Yuko Torigoe, Saki Hayashi, Rie Yoshida, Emiko Goto, Tomoka Konaka, Sayuri Minami (Department of Nursing), Noriko Ishibashi (Medical
Collaboration Center), Kana Kitaguchi, Mikio Ishibashi, Mayumi Iwami, Atsumi Hashimoto (Department of Pharmacy), and Tetsurou Imai (Clinical Engineering center).

CONTRIBUTORS: H.Y. designed the research. H.Y., T.K., S.I., S.Y., T.M., M.I., K.N., R.Y., N.I., A.M., S.F., Y.A. and K.M. performed the study. H.Y. and S.I. and analyzed the data. K.M. and T.T. supervised the project.

\section{REFERENCES}

1. Ministry of Health LaW. Annual Health, Labour and Welfare Report 2013-2014.

2. Panes J, O’Connor M, Peyrin-Biroulet L, Irving P, Petersson $\mathrm{J}$ et al. Improving quality of care in inflammatory bowel disease: what changes can be made today? J Crohns Colitis.

3. Matsumoto T, Yanai S, Toya Y, Ueno M, Nakamura S et al. Internet-orientated Assessment of QOL and Actual Treatment Status in Japanese Patients with Inflammatory Bowel Disease: The 3I survey. J Crohns Colitis 2015; 9(6):477-482.

4. van der Valk ME, Mangen MJ, Leenders M, Dijkstra G, van Bodegraven AA et al. Risk factors of work disability in patients with inflammatory bowel disease--a Dutch nationwide web-based survey: work disability in inflammatory bowel disease. J Crohns Colitis 2014; 8(7):590-597.

5. Hibi T, Panaccione R, Katafuchi M, Yokoyama K, Watanabe $\mathrm{K}$ et al. The 5C Concept and 5S Principles in Inflammatory Bowel Disease Management. J Crohns Colitis 2017; 27;11(11):1302-1308.

6. Swarup N, Nayak S, Lee J, Raikar SP, Hou D et al. Forming a support group for people affected by inflammatory bowel disease. Patient Prefer Adherence 2017; 11:277-281.

7. Langbrandtner J, Huppe A, Jessen P, Büning J, Nikolaus S et al. Quality of care in inflammatory bowel disease: results of a prospective controlled cohort study in Germany (NETIBD). Clin Exp Gastroenterol 2017; 10:215-227.

8. Kim AH, Roberts C, Feagan BG, Banerjee R, Bemelman W et al. Developing a Standard Set of Patient-Centred Outcomes for Inflammatory Bowel Disease - an International, Cross-disciplinary Consensus. J Crohns Colitis 2018; 12(4):408-418. 